\title{
Perioperative Gastrointestinal Care Benefits Surgical Adolescent Idiopathic Scoliosis Patients
}

\author{
Email address: \\ Zengtianyu26@126.com (Haiyan Li) \\ ${ }^{*}$ Corresponding author
}

Yujing Gao, Wenfei Liang, Haiyan Li ${ }^{*}$

The First Affiliated Hospital of Jinan University, Guangzhou, China

\section{To cite this article:}

Yujing Gao, Wenfei Liang, Haiyan Li. Perioperative Gastrointestinal Care Benefits Surgical Adolescent Idiopathic Scoliosis Patients. American Journal of Nursing Science. Vol. 7, No. 2, 2018, pp. 54-57. doi: 10.11648/j.ajns.20180702.12

Received: January 31, 2018; Accepted: February 16, 2018; Published: March 20, 2018

\begin{abstract}
In order to evaluate the effect of perioperative gastrointestinal care on adolescent idiopathic scoliosis (AIS) patients after correction of scoliosis surgery, 13 cases of AIS patients from January 2015 to February 2018 were treated with perioperative gastrointestinal management. The effect was compared to those 11 routine nursing surgical AIS patients from January 2008 to December 2014. Data analysis regarding the age and scoliosis degree showed no statistically significant difference between two groups $(\mathrm{P}>0.05)$. However, abdominal distension degree, bowel sound recovery time and anal exhaust time of the perioperative gastrointestinal care patients were better than those of normal nursing patients $(\mathrm{P}<0.05)$. Furthermore, evaluation of the intestinal function including fixed independent evaluation and evacuation time also was high as compared to those of normal nursing patients. In conclusion, perioperative gastrointestinal care is beneficial to surgical AIS patients.
\end{abstract}

Keywords: Adolescent Idiopathic Scoliosis, Perioperative Nursing, Gastrointestinal Care, Surgery

\section{Introduction}

Adolescent idiopathic scoliosis (AIS) is the most common form of scoliosis and typically affects children. Idiopathic scoliosis affects $2-3 \%$ of children aged $10-18$ years old and is more common among females than males. In idiopathic scoliosis, the specific cause is unknown; however it is largely thought to be a genetic disorder. Most patients with adolescent idiopathic scoliosis have small curves $\left(10 \circ\right.$ to $\left.20^{\circ}\right)$ that do not require formal treatment. Curves that are $30^{\circ}$ to $35^{\circ}$ will likely progress into adulthood and can cause increasing back pain, accelerated disc degeneration, deformity and eventually problems with heart and lung function. Surgery for adolescent idiopathic scoliosis is usually necessary for patients whose curve is larger than $40^{\circ}$ to 45 degrees [1]. In these cases, surgery is the best option to prevent further progression of the curvature. However, scoliosis surgery has a varying but high rate of complications. In the Scoliosis Research Society Morbidity and Mortality Reports, 57 of all patients with spinal fusion $(0.2 \%)$ died mainly due to cardiac causes, 59 of the scoliosis patients $(0.8 \%)$ had neurological deficits, infections between $0.9 \%$ and $3.4 \%$ and an overall complication rate of $8.6 \%$ when all scoliosis are concerned [2]. Among these complications, the incidence of gastrointestinal complications is very high, but these are often overlooked [3]. Gastrointestinal dysfunction caused by correction of scoliosis surgery would lead to gastrointestinal sensorimotor disorders.

The gastrointestinal function recovery of the surgical patients often requires a certain time [4]. The postoperative abdominal distention, intestinal obstruction and other complications were caused by the delayed gastrointestinal function recovery, which affects the quality of life of the patient, prolongs the hospitalization time of the patient, increases the nursing workload, and even needs to be operated again. Therefore, how to safely, effectively promote the recovery of gastrointestinal function and prevent postoperative complications has been widely concerned by medical staff. In recent years, with the deepening of the concept of rapid rehabilitation, an effective gastrointestinal program and effective evacuation should deal with the issues of poor appetite and constipation, and then we can prevent complications [5]. The objective of the current study was to evaluate the perioperative gastrointestinal care management applied to 13 individuals with ASI after surgery in hospital 
from Year 2008 to 2018, when comparing with 11 individuals by normal nursing.

\section{Materials and Methods}

\subsection{Subject Selection}

Thirteen patients with ASI from Jan, 2015 to Feb, 2018 in the First Affiliated Hospital of Jinan University were selected as treatment group, containing 10 girls and 3 boys with average age $15.8 \pm 6.2$. Eleven ASI surgical patients during Jan, 2008 to Dec, 2014 with routine nursing were selected into control group by a retrospective analysis, including 8 girls and 3 boys with average age $15.5 \pm 1.9$. The lenke type of scoliosis and scoliosis degree were very similar in both groups (specific data in Table 1). Inclusion criteria: the AIS patients get spinal surgery; no original gastrointestinal disease; patient or his/her caregiver willing to cooperate.

\subsection{Perioperative Gastrointestinal Care}

Preoperative nursing: The AIS patients in two groups were carried out nursing education after admission, and they have to fast for 8 hours and forbid drinking for 4 hours before operation. In addition, in treatment group, we guided patients to eat light, easy to digest soft food, avoid eating raw, cold, spicy and sweet food, milk, soy milk and other gas-producing foods. Furthermore, Patients were trained 3 days before operation to defecate in bed to prepare for postoperative bed rest.

Postoperative nursing: The AIS patients in two groups were asked to drink a small amount of water and feed the spoon meat after exhausting anus. After 3 days, a normal diet was restored. Thirteen individuals in treatment group underwent standardized bowel training which include five steps [6]. A table was developed to record the bowel situation; the training was last for 2 weeks to observe the best bowel method for each patient, then other methods were taught. Patients in control group were nursed by routine care, including cleaning care to independent evacuation patients; laxatives given and dietary guidance when constipation happened; skin care and antidiarrheal treatment when diarrhea happened.

\subsection{Observing Index}

Two groups of postoperative ASI patients were observed with abdominal distension degree, bowel sound recovery time and the first anal exhaust time. (1) Abdominal distension degree: 5 points are full score, and no abdominal distention is 0 score (patient without abdominal distension, check body abdomen flat soft). Mild abdominal distention is 1 point (The patient complained that there is gas rotation in the abdominal cavity, have mild abdominal distention feeling. Patient's abdominal muscle tension is mild). Moderate abdominal distension are 3 points (between mild and severe abdominal distension), and severe abdominal distension are 5 points (patient complained incision flatulence, abdominal distension feeling obvious). The degree of abdominal distension was observed at 6 hours after operation. After that we record it every 4 hours until the patient exhaust anal, and the score was recorded according to the mean value. (2) Bowel sound recovery time: we do the auscultation for 1 minute in the abdominal every 2 hours after operation, if there are 3 or more bowel sounds, it means the gastrointestinal function is normal. (3) The first anal exhaust time: We inform patients and their families about the phenomenon of anal exhaust before surgery, so that they can report the first exhaust time correctly in time. And we took some other effective and measurable observation indexes including (4) Independent evacuation, fixed evacuation every day or every other day by rectal irritation or laxatives, with soft defecate; (5) The times of evacuation less than $30 \mathrm{~min}$.

\subsection{Statistical Analysis}

Age, degree of curve and other indexes between the treated group and control group were analyzed by SPSS 21.0. The statistical significance of the differences was analyzed using Student's t-test between two groups. $\mathrm{P}<0.05$ was considered to be statistically significant.

\section{Results}

All patients were divided into gastrointestinal care group (Treatment group) and normal nursing group (Control group) according to this study. Age and curve degree were evaluated between the gastrointestinal care group and control group. Statistical data were presented as mean \pm SD. $\mathrm{P}<0.05$ was considered as significant difference. The results shown in Table 1 indicated that the AIS patients between the gastrointestinal care group and control group had no significant differences.

Table 1. Patient information.

\begin{tabular}{|c|c|c|c|c|c|c|c|c|}
\hline \multirow{2}{*}{ Group } & \multirow{2}{*}{ Number of cases } & \multirow{2}{*}{ Age (years) } & \multicolumn{2}{|c|}{$\operatorname{Sex}(n)$} & \multicolumn{3}{|c|}{ Lenke type (n) } & \multirow{2}{*}{ Curve degree Cobb angle $\left(^{\circ}\right)$} \\
\hline & & & Male & Female & $\mathbf{I}$ & II & III & \\
\hline Gastrointestinal care group & 13 & $15.8 \pm 6.2$ & 3 & 10 & 2 & 1 & 10 & $51.3 \pm 10.3$ \\
\hline Control group & 11 & $15.5 \pm 1.9$ & 3 & 8 & 3 & 0 & 8 & $51.0 \pm 8.6$ \\
\hline $\mathrm{t}$ value & & -0.172 & & & & & & -0.078 \\
\hline$P$ value & & 0.866 & & & & & & 0.938 \\
\hline
\end{tabular}

Postoperative intestinal function recovery was comparied as mentioned in the method section. The abdominal distension degree (scores), bowel sound recovery time and the first anal exhaust time were shown in Table 2. Statistical data were presented as mean $\pm \mathrm{SD}$. $\mathrm{P}<0.05$ was considered as significant difference. These results showed that gastrointestinal care management significantly reduced abdominal distension score, shortened bowel sound recovery time and the first anal exhaust time. These difference were statistically significant $(\mathrm{P}<0.05$, see Table 2$)$. 
Table 2. Comparison of the postoperative intestinal function recovery.

\begin{tabular}{lllll}
\hline Group & Number of cases & Abdominal distension degree (score) & Bowel sound recovery time (h) & The first anal exhaust time (h) \\
\hline Gastrointestinal care group & 13 & $1.5 \pm 1.1$ & $18.1 \pm 4.8$ & $29.7 \pm 5.0$ \\
Control group & 11 & $3.0 \pm 1.3$ & $25.1 \pm 8.8$ & $38.4 \pm 8.1$ \\
t value & & 3.095 & 2.472 & 3.252 \\
P value & 0.005 & 0.022 & 0.004 \\
\hline
\end{tabular}

Evaluation of intestinal function was performed as mentioned in the method section. Statistical data were presented as mean and the percentage scores in Table 3. These data showed that the standardized bowel training significantly increased independent evacuation by patients themselves, and shortened the time of evacuation $(\mathrm{P}<0.05$, see Table 3$)$.

Table 3. Evaluation of the intestinal function between two groups.

\begin{tabular}{|c|c|c|c|c|}
\hline & Gastrointestinal care group & Control group & t value & P value \\
\hline Fixed independent evaluation [n (\%)] & $12(92.3 \%)$ & $5(45.5 \%)$ & -4.053 & 0.001 \\
\hline Evacuation less than $30 \mathrm{~min}[\mathrm{n}(\%)]$ & $11(84.6 \%)$ & $3(27.3 .2 \%)$ & 3.384 & 0.003 \\
\hline
\end{tabular}

\section{Discussion}

This study revealed that after perioperative gastrointestinal care, gastrointestinal functions including abdominal distension degree, bowel sound recovery time, the first anal exhaust, incontinence and evacuation time of the surgical AIS patients were largely relieved or shortened, as compared to regular nursing care AIS patients. Furthermore, the evacuation way (referred to fixed independent evaluation and evacuation time) was close to normal life, indicating the successful achievement of perioperative gastrointestinal care of surgical AIS patients.

The aim of the surgical treatment for AIS is to correct the angle of scoliosis and try to keep the physiological equilibrium of the spine [7]. However, spinal surgery often brings a lot of complications to the patient, and the overall major complication rate was $5.4 \%$, of which the complications of the gastrointestinal tract are often overlooked [2]. A lack of priority and awareness from staff regarding bowel care was identified in the baseline focus group. The ICU bowel care often seems to assume low priority [8, 9]. Constipation is a major gastrointestinal complication in the surgical or critically ill patient and the reported incidence of constipation in the general population is between $2 \%$ and $25 \%[8,10]$. In addition, there are numerous complications associated with constipation in the critically ill patient, management of constipation and diarrhoea may be improved by the use of a standardised protocol and careful attention [11]. Furthermore, as reported, standardization bowel management intervention is beneficial to the patients with spinal cord injury to form regular bowel habit [6]. The findings of those studies are similar to this study.

Gastrointestinal dysfunction would lead to a series of problems such as prolong the hospitalization time of the patient, increase the nursing workload, promote mental stress and other psychological pressure and even need to be operated again. Obviously, the early intervention with perioperative gastrointestinal care would largely contribute to the ordered and regular evacuation of surgical AIS patients, and finally benefit the life quality of these AIS patients.

\section{Conclusion}

The quick rehabilitation of gastrointestinal function is important for the quantified life of AIS patients after spinal surgery. Perioperative gastrointestinal care is beneficial to form regular bowel habit for those adolescent idiopathic scoliosis patients who had the spinal surgery, thus improving their life living quantity.

\section{Acknowledgements}

The authors declare no conflict of interest. This work was supported by Medical Scientific Research Foundation of Guangdong Province, China (No. A2017184).

\section{References}

[1] Burton, M. S., Diagnosis and treatment of adolescent idiopathic scoliosis. Pediatr Ann, 2013. 42 (11): p. 224-8.

[2] Weiss, H. R. and D. Goodall, Rate of complications in scoliosis surgery - a systematic review of the Pub Med literature. Scoliosis, 2008. 3: p. 9.

[3] Burton, D. C., et al., Results of the Scoliosis Research Society Morbidity and Mortality Database 2009-2012: A Report From the Morbidity and Mortality Committee. Spine Deform, 2016. 4 (5): p. 338-343.

[4] Wen, Z., et al., Chewing gum for intestinal function recovery after caesarean section: a systematic review and meta-analysis. BMC Pregnancy Childbirth, 2017. 17 (1): p. 105.

[5] Thomas, D. C., et al., Rehabilitation of the patient with chronic critical illness. Crit Care Clin, 2002. 18 (3): p. 695-715.

[6] Liu, C., Standardized Bowel Training Benefits Patients with Neurogenic Bowel Dysfunction After Spinal Cord Injury. American Journal of Nursing Science, 2015. 4 (6): p. 305.

[7] Rodrigues, L. M. R., et al., Adolescent idiopathic scoliosis: surgical treatment and quality of life. Acta Ortop Bras, 2017. 25 (3): p. 85-89. 
[8] McPeake, J., H. Gilmour, and G. MacIntosh, The implementation of a bowel management protocol in an adult intensive care unit. Nurs Crit Care, 2011. 16 (5): p. 235-42.

[9] Dorman, B. P., et al., Bowel management in the intensive care unit. Intensive Crit Care Nurs, 2004. 20 (6): p. 320-9.
[10] Ramkumar, D. and S. S. Rao, Efficacy and safety of traditional medical therapies for chronic constipation: systematic review. Am J Gastroenterol, 2005. 100 (4): p. 936-71.

[11] Mostafa, S. M., et al., Constipation and its implications in the critically ill patient. Br J Anaesth, 2003. 91 (6): p. 815-9. 